\title{
Optical Forces Induced by Metal Nanoparticle Clusters
}

\author{
Jordi Sancho-Parramon ${ }^{1,2 *}$, Salvador Bosch ${ }^{2}$ \\ ${ }^{1}$ Rudjer Boskovic Institute, Zagreb, Croatia \\ ${ }^{2}$ Applied Physics and Optics Department, University of Barcelona, Barcelona, Spain \\ *corresponding author, E-mail: j sancho@irb.hr
}

\begin{abstract}
The strong field localization generated between closely placed metal particles excited by electromagnetic radiation induces intense forces on small polarizable objects. In this study we investigate the optical forces that can be generated in the vicinity of metal nanoparticle clusters using fully electrodynamic numerical simulations. The influence of the cluster configuration as well as of the excitation parameters is analyzed.
\end{abstract}

\section{Introduction}

Optical trapping appears to be a promising way to control the position of small objects with nanometric precision. In this sense, optical tweezers have been shown to be a useful tool to trap objects in the focal region of tightly focused beams [1]. The key issue is the gradient of electromagnetic field generated at the focus that induces a dipole force on polarizable particles. This force, proportional to the field intensity gradient, drives the particles towards the region where the field is largest. However, due to the diffractionlimited field distribution that can be achieved with conventional optics, nanometric control of the position of small objects requires large laser power beams. Such requirement is not suitable for objects that might be damaged when exposed to strong irradiation, for instance, biological samples.

In order to surpass this restriction, the use of optical near-fields for trapping has been proposed as alternative, since near-field distributions are not diffraction-limited. In this sense nanometric optical tweezers have been suggested for precise trapping of very small objects based on the strong field gradient generated around nanoparticle surfaces [2]. Using this principle, nanoscale control of objects has been experimentally demonstrated at nanostructured substrates containing metal particle pairs $[3,4]$ enabling even stacking and sorting of objects [5]. Such trapping configurations exploit the strongly enhanced and localized near field generated at the gap between particles as result of the coupling of individual particle plasmon resonances [6].

In the present study we analyze the potential of metal clusters for efficient optical trapping of small objects. In particular, we focus on the influence of different configurations of particle pairs and of the characteristics of the radiation exciting the cluster. The evaluation of the potential for trapping for each configuration is performed by calculating the near field distribution in the cluster structure. For this purpose we use fully electrodynamic simulations, i.e., a generalized Mie theory that can take into account different particle clusters and illumination conditions. The numerical simulations evidence how the trapping potential of focused beams can be boosted by the presence of metal nanoparticles in the focal region and aid in choosing the best particle configurations for optimal trapping.

\section{Theory}

A general sketch of the system investigated is shown in Fig. 1 . We assume a plane wave travelling in the $-z$ direction with a wave-vector $(0,0,-k)$ and focused by an aplanatic lens with numerical aperture NA $=n_{m} \cdot \sin \alpha$ where $n_{m}$ is the refractive index in the image space and $\alpha$ is the angular semi-aperture of the lens. The presence of a cluster of small particles and an object to be trapped in the focal region may significantly modify the incident electromagnetic field distribution. The focus of the system is located at $x=y=z=$ 0 . In order to evaluate how an object can be trapped it is necessary to compute: i) the field distribution of the focused plane wave, ii) the electromagnetic response of the cluster of particles and the object to be trapped to this incident field distribution, and iii) the force exerted by the resulting total field distribution on the object to be trapped.

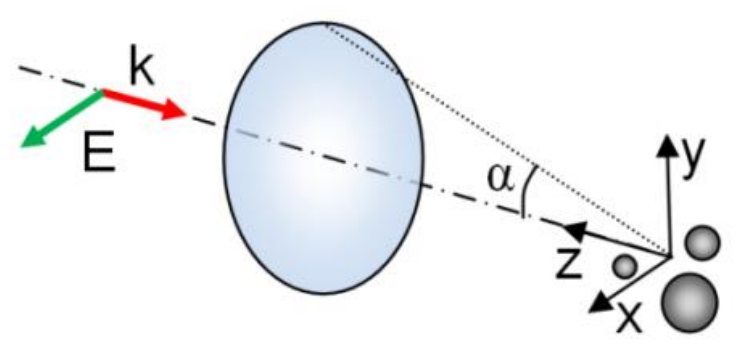

Figure 1: Scheme of the system considered in the numerical simulations. A cluster of particles located in the focal region of a lens is excited by the incident focused beam. 


\subsection{Focal field distribution}

The distribution of light in the focal region of a high numerical aperture lens can be calculated using the Richards-Wolf theory [7]. Imposing the aplanatism condition, the electric field at a point $\mathbf{r}$ in the focal region, $\mathbf{E}_{\mathbf{f}}$, can be computed with the following diffraction integral:

$\mathbf{E}_{\mathbf{f}}(\mathbf{r})=\frac{-i}{l} \check{\mathbf{n}}_{0}{ }^{a} d q \sin q \check{\mathbf{n}}_{0}{ }^{2 p} d f \mathbf{E}_{r e f} \exp (-i \mathbf{k} \times \mathbf{r})$,

where $\lambda$ is the vacuum wavelength and $\mathbf{E}_{r e f}$ is the field refracted by the lens that can be calculated from the incident plane wave by geometrical optics. $\mathbf{k}$ is a wavector with module $k$ and with direction given by $(\theta, \phi)$, that are the polar and azimuthal angles in a spherical coordinate system. Actually, the above diffraction integral is the angular spectrum representation of the focal field, i.e., the field at focus can be interpreted as a sum of plane waves with polarizations and weights determined by the propagation direction [8].

\subsection{Electromagnetic response of particles}

The interaction of a cluster of arbitrary sized and shaped particles with electromagnetic radiation is a complex problem that usually can be only solved by numerical resolution of Maxwell equations. However, if the particles of the cluster and the object to be trapped are spherical, the solution can be found in a in a semi-analytical way using the generalized Mie theory [9]. Briefly, the incident, scattered and internal fields are expanded in vector spherical harmonics, that are combination of spherical harmonics and Bessel functions. Then, imposing boundary conditions at the surface of every particle, the expansion coefficients of the scattered and internal fields can be calculated from those of the incident field by solving a linear system of equations. In order to solve this system of equations is necessary to use vector addition theorems that connect vector spherical harmonics with different origins, i.e., particle centers. The dimension of the system of equations is determined by the number of terms in the field expansion. Therefore, the higher accuracy is required, the larger the size of the system of equations and thus, the computational load.

The expansion coefficients for an incident plane wave are well known [9]. Generally speaking, for an arbitrary field distribution is always possible to find the expansion coefficients numerically [10]. Nevertheless, for a focused field distribution given by Eq. 1, different authors have proposed multipole expansions of the diffraction integral for linearly polarized plane waves [11] or cylindrical vector beams [12] illuminating the lens. These expansions can be easily related to the vector spherical harmonics expansion used in the generalized Mie theory. We have recently used such expansions to analyze plasmon coupling in tightly focused beams [13-14].

\subsection{Calculation of forces and related parameters}

Once the electromagnetic field distribution is known, the time-averaged force acting over an object is given by [8]:

$$
\langle\mathbf{F}\rangle=\int_{S}\langle\mathbf{T}\rangle \cdot d \mathbf{s}
$$

where $\langle\overrightarrow{\mathbf{T}}\rangle$ is the time-averaged Maxwell stress tensor and the integral is performed over a surface enclosing the object. The Maxwell stress tensor can be directly calculated from the electromagnetic field as:

$$
\ddot{\mathbf{T}}=\left[\varepsilon_{m} \mathbf{E} \otimes \mathbf{E}+\mu_{m} \mathbf{H} \otimes \mathbf{H}-\frac{1}{2}\left(\varepsilon_{m} E^{2}+\mu_{m} H^{2}\right) \ddot{\mathbf{I}}\right],
$$

where $\mathbf{E}$ and $\mathbf{H}$ stand for the electric and magnetic fields, $E$ and $H$ are their magnitudes and $\varepsilon_{m}$ and $\mu_{m}$ the medium permittivity and permeability. Alternatively, a simplified computation framework can be used if the particle to be trapped is much smaller than the wavelength and the field acting over the particle can be locally approximated to a plane wave. If, in addition, the particle is non-absorbing, the average force can be calculated as [15]:

$$
\langle\mathbf{F}\rangle=\frac{\alpha n_{m}}{2 c} \nabla I_{e x c},
$$

where $c$ is the speed of light in vacuum, $I_{\text {exc }}$ is the intensity of the electromagnetic field exciting the particle (i.e., without the presence of the particle) and $\alpha$ is the particle polarizability, that can be calculated from the ClausiusMossotti relation:

$$
\alpha=3 V \frac{\varepsilon_{p}-\varepsilon_{m}}{\varepsilon_{p}+2 \varepsilon_{m}},
$$

with $V$ being the particle volume and $\varepsilon_{p}$ the particle permittivity. Equation 4 represents the gradient force and states that small particles are driven to the regions with largest field intensity. Around this equilibrium position, the restoring force exerted on the particle has a linear dependence with small displacements $\Delta x$, enabling to define the trap stiffness as:

$$
k_{\text {trap }}=-\frac{\langle\mathbf{F}\rangle}{\Delta x}
$$

In addition, an important parameter to quantify the stability of the optical trap to Brownian motion is the trapping potential, defined as the work that has to be done to bring a particle from a given position ro to infinity:

$$
U\left(\mathbf{r}_{\mathbf{0}}\right)=-\int_{\infty}^{\mathbf{r}_{0}}\langle\mathbf{F}\rangle \cdot d \mathbf{r}
$$

Finally, a convenient way to quantify how effective is a given illumination configuration for work as an optical trap is the trapping efficiency, $\mathbf{Q}$, defined as:

$$
\mathbf{Q}=\langle\mathbf{F}\rangle \frac{c}{n_{m} P_{i n c}}
$$

where $P_{\text {inc }}$ is the power of the incident beam. 


\section{Results and discussion}

In the following we shall assume that the radiation illuminating the lens is a plane wave linearly polarized in the $x$-direction and with $\lambda=1064 \mathrm{~nm}$. The power incising on the lens is set to $100 \mathrm{~mW}$. The object to be trapped is a small sphere with radius $1 \mathrm{~nm}$ and refractive index 1.5. The medium in the image space is assumed to be water $\left(n_{m}=\right.$ 1.33). For the cluster of particles we consider $\mathrm{Au}$ with complex refractive index $0.26+6.97 \mathrm{i}$ at the radiation wavelength. We observed that for all the studied cases, there are no significant differences when the force acting on the glass particle is calculated either by Eq. 2 or Eq. 4 .

\subsection{Focused beam trapping vs. plasmon field trapping}

First, we aim to illustrate the enhancement of trapping by a focused beam if the strong near field associated to the plasmon resonance is used. Fig.2 shows the intensity of the incident beam at the focal plane (left) for a lens with $\mathrm{NA}=$ 1.2 and the total field when two gold particles with radius $R$ $=80 \mathrm{~nm}$ and interparticle distance $d=20 \mathrm{~nm}$ are located over the $x$-axis, i.e., at $x= \pm 90 \mathrm{~nm}, y=z=0$. The strong electromagnetic coupling between the particles [13] results in charge accumulation in the zones where the particles are facing each other and the formation of a hot-spot, i.e., region of nanometric dimensions and a large field enhancement (over two orders of magnitude with respect to the incident field intensity for the given configuration).
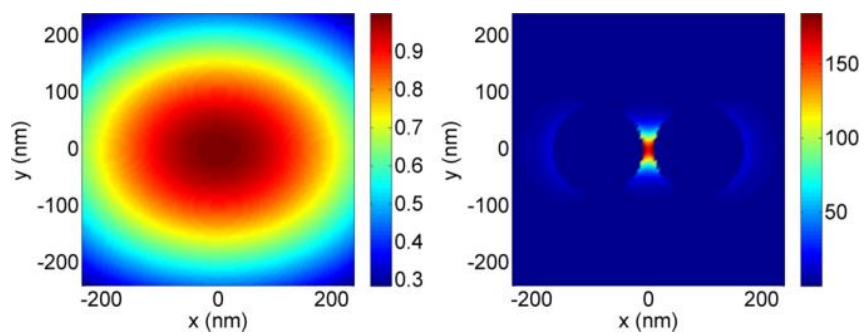

Figure 2: Intensity (a.u.) distribution at the focal plane for the incident focused beam (left) and with the presence of the Au particles (right).

The large intensity gradient that can be achieved by plasmon coupling compared to the one that can be attained by conventional focusing gives place to much stronger forces, as shown in Table 1, where several force-related parameters are compared when the glass 1-nm particle is located over the $y$-axis of the focal plane.

Table 1: Optical trapping parameters for $1 \mathrm{~nm}$ glass particle (see text for details on illumination)

\begin{tabular}{ccc}
\hline & $\begin{array}{c}\text { no } \mathrm{Au} \\
\text { particles }\end{array}$ & $\begin{array}{c}\text { with } \mathrm{Au} \\
\text { particles }\end{array}$ \\
\hline$k_{\text {trap }}(\mathrm{pN} / \mu \mathrm{m})$ & $2.2 \times 10^{-5}$ & 0.32 \\
$Q_{y}($ maximum $)$ & $7.2 \mathrm{e}-9$ & $9.7 \mathrm{e}-6$ \\
$U_{\min }\left(\right.$ in $\left.\mathrm{k}_{\mathrm{B}} \mathrm{T}\right)$ & $-2.87 \mathrm{e}-5$ & -0.0373 \\
$(\mathrm{~T}=300 \mathrm{~K})$ & &
\end{tabular}

\subsection{Illumination conditions}

The influence of the degree of focusing on the trapping efficiency is shown in Fig.3. The configuration is the same as described in the previous section and the only parameter that is changed is the numerical aperture of the system. For a focused beam, weaker focusing leads to lower intensity gradient and, therefore, to smaller optical force. In addition, the position where force is maximal, i.e., where the intensity gradient is largest, varies with the dimension of the focal spot size, that increases as the NA is reduced. This is not the case for the plasmon-based trapping, where the position where the force is maximal is entirely dictated by the spatial extension of the hot-spot. The absolute value of the force also decreases when the NA of the system is lowered. Since the field enhancement is nearly independent of the NA, the force decrease can be basically associated to the lower incident field intensity at the focus when the NA of the system is reduced. Indeed, it appears that tight focusing is not requested to enable trapping of small particles by plasmon coupling, as even under standard illumination conditions, trapping of molecules can take place if the space between metal particles is in the range of few nanometers, leading to a field enhancement of several orders of magnitude [16].
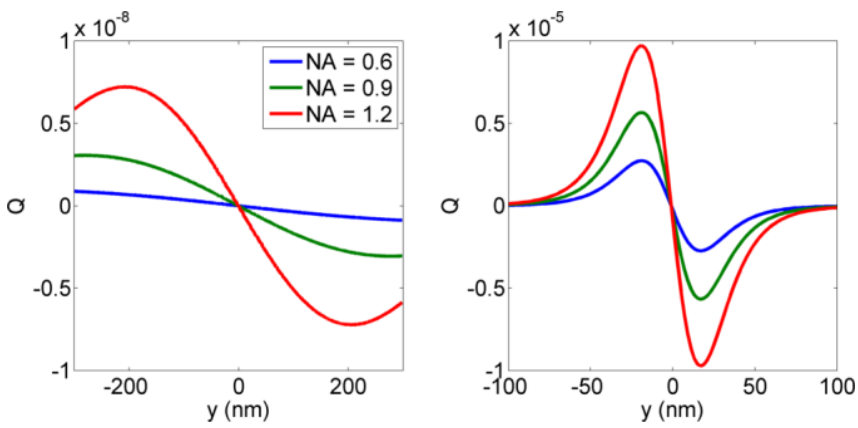

Figure 3: NA dependence of the trapping efficiency for a $1 \mathrm{~nm}$ glass particle located on the $y$-axis of the focal plane without (left) and with (right) the presence of Au particles.

We have also investigated other illumination conditions, such as different linearly polarized plane waves or cylindrical vector beams, but it appears that the largest gradient, and therefore, force, is always achieved through the hot-spot generated by a polarization parallel to the particle pair.

\subsection{Cluster configuration}

Finally, we study the effect or re-scaling the dimensions of the $\mathrm{Au}$ pair of particles. Thus, in addition to the above configuration with particle radius $R=80 \mathrm{~nm}$ and interparticle distance $d=20 \mathrm{~nm}$, we consider also the case with $R=40 \mathrm{~nm}$ and $d=10 \mathrm{~nm}$ and $R=20 \mathrm{~nm}$ and $d=5$ $\mathrm{nm}$, i.e., the whole system is re-scaled a factor 2 and 4 . The wavelength dependence of the magnitude of the field enhancement at the focus (between the particles) is shown in Fig. 4. The maximum of field enhancement is associated to the excitation of the coupled plasmon mode, which shifts 
to longer wavelength the larger the particle. On the other hand, due to radiative damping, large particles give place to a reduced near-field enhancement in comparison with small particles. In addition, decreasing the interparticle distance amplifies the coupling strength and gives place to larger field enhancements [17]. Overall, the maximum field enhancement at the wavelength considered in the present simulations is obtained with the $R=80 \mathrm{~nm}, d=20 \mathrm{~nm}$ configuration.

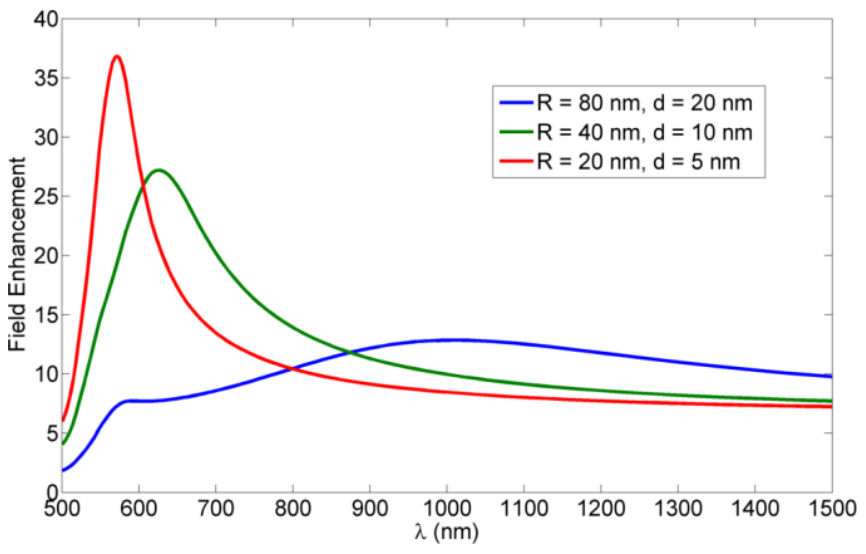

Figure 4: Electric field enhancement at the focus when different $\mathrm{Au}$ particle pairs are located over the $x$ axis of the focal plane ( $x= \pm(R+d / 2), y=z=0)$.

The trapping efficiency and optical trapping potential of these different particle pairs is shown in Fig. 5. Although the field enhancement is lower for smaller particle pairs, the maximum force is larger since the size of the hot spot is reduced and therefore the intensity gradient can be larger than for bigger particles [18]. On the other hand, a smaller spatial extension of the trapping region leads to a narrower, but also smaller in magntiude, optical trapping potential.
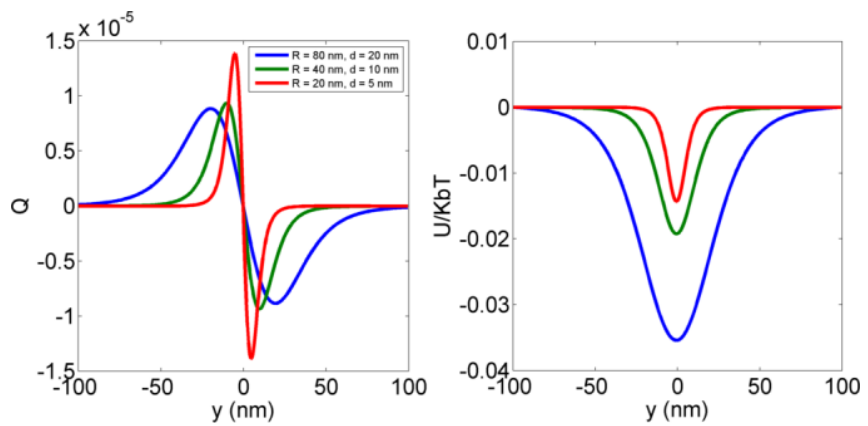

Figure 5: Trapping efficiency (left) and optical trapping potential (right) for different Au particle pairs configurations located over the $x$ axis of the focal plane $(x= \pm(R+d / 2), y=z=0)$.

\section{Conclusions}

The potential of plasmon coupling for trapping of nanometric sized objects has been investigated by numerical simulations. Our approach takes into account with no approximation: i) the description of focused light as field exciting the metal nanoparticles, ii) the modification of this field distribution by the electromagnetic response of the metal nanoparticles and iii) the forces exerted over a small dielectric particle. It is shown that the strong field gradients generated by plasmon coupling and that are confined in nanometric-sized regions lead to optical forces that are several orders of magnitude larger than those that can be obtained in absence of the metal particles. In addition, the influence of the illumination conditions and of the cluster dimensions enables to elucidate what system configurations are more suitable for optimizing the trapping of nanoobjects.

\section{Acknowledgements}

JSP acknowledges the financial support of the Government of Catalonia through a Beatriu de Pinós post-doctoral grant. The authors also acknowledge the support of the project FIS2012-38244-C02-02 of the Spanish Ministry of Economy and Competitivity.

\section{References}

[1] A. Ashkin, Acceleration and trapping of particles by radiation pressure, Phys. Rev. Lett. 24: 156-159, 1977.

[2] L. Novotny, R. X. Bian, and X. S. Xie, Theory of nanometric optical tweezers, Phys. Rev. Lett. 79: 645648, 1970.

[3] A. N. Grigorenko, W. Roberts, M. R. Dickinson, and Y. Zhang, Nanometric optical tweezers based on nanostructures substrates, Nat. Photonics 2: 365-670, 2008

[4] M. Righini, P. Ghenuche, S. Cherukulappurath, V. Myroshnychenko, F. J. García de Abajo, and R. Quidant, Nano-optical Trapping of Rayleigh particles and Escherichia coli bacteria with resonant optical antennas, Nano Lett., 9: 3387-3391, 2009.

[5] B. J. Roxworthy, K. D. Ko, A. Kumar, K. H. Fung, E. K. C. Chow, G. L. Liu, N. X. Fang, and K. C. Toussaint, Jr, Application of plasmonic bowtie nanoantenna Arrays for optical trapping, stacking, and sorting, Nano Lett. 12: 796-801, 2012.

[6] J. R. Krenn, A. Dereux, J. C. Webber, E. Bourillot, Y. Lacroute, J. P. Goudonnet, G. Schider, W. Gotschy, A. Leitner, F. R. Aussenegg, amd C. F. R. Girard, Squeezing the optical near-field zone by plasmon coupling of metallic nanoparticles, Phys. Rev. Lett. 82: 2590-2593, 1999.

[7] B. Richards and E. Wolf, Electromagnetic diffraction in optical systems. II. Structure of the image field in an aplanatic system. Proc. Royal Soc. Lond. 253(1274): 358-379, 1959.

[8] L. Novotny and B. Hecht. Principles of Nano-optics. Cambridge university press, 2006.

[9] J. M. Gérardy and M. Ausloos, Absorption spectrum of clusters of spheres from the general solution of 
Maxwell's equations. II. Optical properties of aggregated metal spheres, Phys. Rev. B 25: 4204-4229, 1982.

[10]C. F. Bohren and D. R. Huffman, Absorption and scattering of light by small particles. Wiley-Vch, 2008.

[11] C. J. R. Sheppard and P. Török, Efficient calculation of electromagnetic diffraction in optical systems using a multipole expansion, J. Mod. Optics 44:803-818, 1997.

[12] R. Borghi, M. Santarsiero, and M. A. Alonso, Highly focused spirally polarized beams, J. Opt. Soc. Am. A, 22:1420-1431, 2005.

[13] J. Sancho-Parramon, Near-field coupling of metal nanoparticles under tightly focused illumination, Opt. Lett. 36:3527-3529, 2011

[14] J. Sancho-Parramon and S. Bosch., Dark modes and Fano resonances in plasmonic clusters excited by cylindrical vector beams, ACS nano 6:8415-8423, 2012.

[15]A. Rohrbach and E. Stelzer, Optical trapping of dielectric particles in arbitrary fields, J. Opt. Soc. Am. A, 18:839-853 (2001).

[16] H. Xu and M. Käll, Surface-plasmon-enhanced optical forces in silver nanoaggregates, Phys. Rev. Lett. 89:246802, 2002.

[17] U. Kreibig and M. Vollmer, Optical properties of metal clusters, Springer Series in Materials Science, 1995.

[18]A. A. E. Saleh and J. A. Dionne, Toward efficient optical trapping of sub-10-nm particles with coaxial plasmonic apertures, Nano Lett. 12:5581-558, 2012. 